\title{
Angebotsstrukturen der ambulanten vertragsärztlichen Versorgung: Deutschland und andere dezentral organisierte Gesundheitssysteme
}

\author{
Structures of Outpatient Medical Care: Germany and Other \\ Decentrally Organized Healthcare Systems
}

(ㄷ) (1) (우)

Autoren

Ursula Hahn ${ }^{1,2}$, Christine Baulig ${ }^{1}$, Patrick Brzoska ${ }^{3}$

Institute

1 Lehrstuhl für Medizinische Biometrie und Epidemiologie, Universität Witten/Herdecke Fakultät für Gesundheit, Witten, Deutschland

2 OcuNet GmbH \& Co. KG, Düsseldorf, Deutschland

3 Lehrstuhl für Versorgungsforschung, Universität Witten/ Herdecke Fakultät für Gesundheit, Witten, Deutschland

\section{Schlüsselwörter}

Angebotsstrukturen, Ambulante Versorgung, Ambulante Versorgungsorganisationen, Einzelpraxis, Gemeinschaftspraxis, Medizinisches Versorgungszentrum (MVZ)

\section{Key words}

Delivery system, outpatient health organization, solo practice, group practice, outpatient medical care, medical care centers

online publiziert $\quad 25.03 .2021$

\section{Bibliografie}

Gesundheitswesen 2021; 83: 337-344

DOI 10.1055/a-1390-3935

ISSN $0941-3790$

(C) 2021. The Author(s).

This is an open access article published by Thieme under the terms of the Creative Commons Attribution-NonDerivative-NonCommercial-License, permitting copying and reproduction so long as the original work is given appropriate credit. Contents may not be used for commercial purpose, or adapted, remixed, transformed or built upon. (https://creativecommons. org/licenses/by-nc-nd/4.0/)

Georg Thieme Verlag KG, Rüdigerstraße 14,

70469 Stuttgart, Germany

\section{Korrespondenzadresse}

Ursula Hahn

Lehrstuhl für Medizinische Biometrie und Epidemiologie Fakultät für Gesundheit der Universität Witten/Herdecke Alfred-Herrhausen-Straße 50

58448 Witten

Deutschland

Ursula.Hahn@uni-wh.de

\section{ZUSAMMENFASSUNG}

Hintergrund Das Bild vertragsärztlicher Versorgung ist arztbezogen; wenig beachtet wird der (potentielle) Einfluss von Versorgungsorganisationen (VO) und deren Merkmalen auf das Versorgungsgeschehen.

Ziel Beschreibung der organisationsbezogenen Angebotsstrukturen nach Rechtsformen, Abgleich mit denen anderer dezentral organisierter Gesundheitssysteme und Diskussion, ob sich entlang der Rechtsform Effekte für verschiedene Dimensionen des Versorgungsgeschehens zeigen lassen.

Material und Methoden Die Häufigkeiten von Einzelpraxis, Gemeinschaftspraxis und Medizinischen Versorgungszentren (MVZ) für haus- und fachärztliche Versorgungsbereiche, die durchschnittliche ärztliche Teamstärke und der Anteil der VO mit interdisziplinärer Aufstellung werden auf Basis von Sekundärdatenquellen (überwiegend Kassenärztliche Bundesvereinigung) im Zeitablauf beschrieben.

Ergebnisse Die Angebotsstrukturen sind durch hohe Fragmentierung (2018: > 80000 VO) mit hohem Anteil von Kleinstorganisationen (über alle VO 73 \% Einzelpraxen), niedriger durchschnittlicher Teamstärke (über alle VO 1,7 Ärzte) und geringem Anteil mit interdisziplinärer Aufstellung (17\% aller Gemeinschaftspraxen und MVZ) bei geringer Konzentrationsdynamik in der haus- und fachärztlichen Versorgung geprägt. Schlussfolgerungen Die Kleinteiligkeit der deutschen Angebotsstrukturen ist ausgeprägter bzw. persistierender als die in anderen dezentralen Gesundheitssystemen. Rechtsformen und Durchschnittswerte erfassen die Heterogenität der VO-Morphologien nur unzureichend. Wünschenswert, z. B. für eine gezielte Versorgungssteuerung, wäre eine breitere Datenbasis zu mehr organisationsbezogenen Merkmalen.

\footnotetext{
ABSTRACT

Background The image of outpatient medical care is physiciancentered; little attention is paid to the (potential) influence of health organizations and their characteristics on medical care dimensions. Aim Description of the structures of outpatient medical care stratified by legal forms, comparison with those of other decentralized health systems and discussion of the potential effect of legal form on different dimensions of medical care.
} 
Methods The frequencies of solo practice, group practice and Medical Care Centers for both primary and specialist medical care, the average team size of practices and the proportion of interdisciplinary health organizations are described over time based on secondary data sources (mainly provided by the National Association of Statutory Health Insurance Physicians).

Results The structures of outpatient medical care are characterized by high fragmentation (2018: > 80,000 health organizations), high proportion of small organizations ( $73 \%$ of all health organizations are solo practices), low average team size (1.7 physicians on average) and low proportion of interdiscip- linary health organizations ( $17 \%$ of all group practices and Medical Care Centers). Overall, there is little indication that family and specialist care facilities tend to merge with each other.

Conclusions The fragmentation of the German health care structure is more pronounced and lasting than in other decentralized health systems. Legal forms and average values do not adequately capture the heterogeneity of organizations in outpatient health care. In order to improve organization of medical care, a broader database including more organizational characteristics would be desirable.

\section{Einleitung}

Das Bild der vertragsärztlichen niedergelassenen Versorgung in Deutschland wird wesentlich von dem tätigen Facharzt/der Fachärztin geprägt. Vertragsärztliche Versorgungsorganisationen (VO), also Praxen oder Medizinische Versorgungszentren (MVZ), sind hingegen selten im Fokus, obwohl die Diskussion immer wieder um den arztunabhängigen Einfluss auf das Versorgungsgeschehen kreist [1]. So wird davon ausgegangen, dass gemeinschaftliche Berufsausübung von Ärzten/Ärztinnen verschiedener Fachgruppen innerhalb von Gemeinschaftspraxen oder MVZ im Vergleich zur Versorgung durch Einzelpraxen grundsätzlich bessere Voraussetzungen bei komplexen patientenbezogenen Versorgungsbedarfen bietet [2]. Diskutiert wird auch, dass die Trägerschaft Einfluss auf Art und Umfang des Versorgungsangebots haben kann. Von bestimmten Rechtsformen wird eher eine Versorgung in ländlichen Gegenden erwartet [3]. VO könnte damit grundsätzlich eine eigenständige Qualität in der Beschreibung und Bewertung des Versorgungsgeschehens und der Leistungsfähigkeit von Gesundheitssystemen zukommen.

Als Annäherung an diesen Themenkomplex befasst sich dieser Beitrag mit der strukturierten Erfassung der organisationsbezogenen Angebotsstrukturen im Zeitablauf auf Basis verfügbarer Sekundärdatenquellen. Diese erlauben eine Auswertung nach Rechtsformen und hier nach Anzahlen, durchschnittlicher Teamstärke und interdisziplinärer Aufstellung insgesamt und hausärztlichem und fachärztlichem Versorgungsbereich (im Folgenden „Bereiche“). Der Vergleich mit Angebotsstrukturen anderer dezentraler Gesundheitssysteme dient als Orientierung zur Identifikation von Spezifika des deutschen Systems. Inwieweit diese per öffentlich zugänglichen Statistiken abgreifbaren Merkmale geeignet sind, die Heterogenität in den Angebotsstrukturen abzubilden, wird abschließend diskutiert.

\section{Methode}

Es wurden die folgenden Sekundärdatenquellen verwendet; die Daten wurden z.T. kombiniert ausgewertet und dazu bearbeitet. Quellenbedingte Limitationen bei der Auswertung werden im Folgenden jeweils benannt.

- Statistik der Kassenärztlichen Bundesvereinigung (KBV) „Anzahl Praxen nach Praxisart, 2008-2018“ [4]

- KBV Statistik „Anzahl Ärzte in Praxen nach Art der Praxis, 2010-2018“" [5]
- KBV Statistik „Statistische Informationen aus dem Bundesarztregister“, Tabelle 5 [6],

VO-Typen werden nach Rechtsform (also Einzelpraxis (EP), Gemeinschaftspraxis (GP) und Medizinische Versorgungszentren (MVZ)) sowie nach hausärztlichem (HV) bzw. fachärztlichem Versorgungsbereich (FV) nach § 73 Sozialgesetzbuch V (SGB V) unterschieden. Die Auswertung umfasst nur ambulante vertragsärztliche VO inklusive MVZ in Krankenhausträgerschaft. Ermächtigungsambulanzen wurden nicht einbezogen, da sie üblicherweise keine geschlossene organisatorische Einheit sind. Vertragspsychologische VO und Mischorganisationen (vertragspsychologische und -ärztliche GP) wurden ausgeschlossen, da dazu internationale Vergleichsdaten fehlen.

\section{Anzahl Versorgungsorganisationen}

Die absolute und relative Häufigkeit von VO (Quelle [4]) wird für 2010, 2014 und 2018 ausgewiesen. Nach HV und FV getrennt können nur Anzahlen von EP und GP nicht jedoch von MVZ ausgewiesen werden.

\section{Ärztliche Teamstärke}

Die durchschnittliche ärztliche Teamstärke wird als Quotient aus Anzahl der tätigen Fachärzte/-ärztinnen (Quelle [5] und Anzahl Praxen nach Praxisart (Quelle [4]) für 2010, 2014 und 2018 ermittelt. Für die Auswertung nach Bereichen wurden die (fachgruppenspezifischen) Daten aus Quelle [5] bereichsspezifisch aufsummiert. Zur HV gehören Fachärzten/-innen für Allgemeinmedizin, hausärztlich tätige Internisten/-innen und Fachärzte/-innen für Kinderheilkunde.

\section{Interdisziplinäre Aufstellung: Fachübergreifende und bereichsübergreifende Versorgungsorganisationen}

Fachübergreifende Aufstellung beschreibt die gemeinschaftliche Berufsausübung von Ärzten/-innen aus mindestens 2 Fachgruppen, bereichsübergreifende Aufstellung beschreibt die gemeinschaftliche Berufsausübung von Ärzten/-innen von HV und FV. Im Folgenden wird für beide Merkmale der Begriff „interdisziplinäre Aufstellung“ verwendet, sie ist rechtsformbedingt nur in GP und MVZ möglich.

Die Häufigkeit interdisziplinärer GP wurde der KBV-Quelle [4] entnommen. Die Angaben zu MVZ entstammen einer Antwort der Bundesregierung [7]; diese Daten liegen nur für 2015 bis 2017 vor, daher umfasst die Auswertung nur diesen Zeitraum. 


\section{Ergebnisse}

\section{Angebotsstrukturen in der deutschen vertragsärztlichen Versorgung}

2018 wurde die vertragsärztliche Versorgung in Deutschland von insgesamt 80811 unabhängigen VO verantwortet ( $\triangleright$ Tab. 1). Im Zeitablauf seit 2010 lässt sich ein leichter Trend zur Konzentration erkennen; pro Jahr nahm die Zahl der VO im Schnitt um knapp 1000 ab. 2018 entfielen nahezu drei Viertel aller VO auf EP, sie dominierten trotz leicht rückläufiger Anzahl die Angebotsstrukturen über den gesamten Auswertungszeitraum. Etwas weniger als ein Viertel aller VO entfielen auf GP, ihre Zahl war im Zeitablauf ebenfalls rückläufig. Die Zahl der MVZ stieg von einem niedrigen Ausgangswert absolut wie relativ.

In der HV und FV waren über den gesamten Betrachtungszeitraum in etwa die gleiche Zahl von EP bzw. bereichsinternen GP tätig ( Tab. 1).

\section{Ärztliche Teamstärke}

Rund die Hälfte aller Ärzte/-innen arbeitete 2018 in GP oder MVZ, der Anteil der in EP tätigen Mediziner/-innen war leicht rückläufig ( Tab. 3). Die durchschnittliche ärztliche Teamstärke über alle VO stieg von 2010 bis 2018 von 1,4 auf 1,7 ( Tab. 2). In EP blieb sie weitgehend konstant (2010: 1,1, 2018: 1,2 (Teamstärke in EP> 1, da Inhaber andere Ärzte/-innen anstellen können)), in GP wuchs sie leicht (2010: 2,5, 2018: 2,8). In MVZ stieg sie ausgehend von dem vergleichsweise hohen Ausgangswert von 5,0 Ärzten/-innen auf 6,2 bis 2014, anschließend nahm die durchschnittliche Teamstärke wieder ab und lag 2018 bei 5,9.

Nach Zahl tätiger Fachärzte/-innen ist die FV stärker gewachsen als die HV ( $>$ Tab. 3). Der Anteil der in EP tätigen Ärzte/-innen in der HV lag 201811 Prozentpunkte über dem in der FV, zugleich lag der Anteil der in MVZ tätigen hausärztlichen Fachärzte/-innen mit $6 \%$ deutlich unter der in der FV (20\%). Die EP-Teamstärken waren in HV und FV über den gesamten Auswertungszeitraum nahezu identisch, in fachärztlichen GP arbeiteten im Schnitt mehr Ärzte/innen zusammen als in hausärztlichen ( $\triangleright$ Tab. 2).

Auf die durchschnittliche Teamstärke wirkten 2 Einflussfaktoren: Die VO-Gesamtzahl ging um $9 \%$ zurück ( $>$ Tab. 1 ), gleichzeitig stieg die Gesamtzahl der vertragsärztlichen Mediziner/-innen um $9 \%$ ( $>$ Tab. 3 ).

\section{Interdisziplinäre Aufstellung von Versorgungsorganisationen}

2017 waren insgesamt 3686 GP und MVZ interdisziplinär aufgestellt, das entspricht $17 \%$ dieser VO (8\% der GP und $75 \%$ der MVZ) ( Abb. 1). Von den interdisziplinären GP waren 642 fachübergreifende und 772 bereichsübergreifende GP. Absolut wie relativ entwickelte sich die Zahl interdisziplinärer GP stetig rückläufig, besonders stark bei den bereichsübergreifenden GP (in 2010 noch 1136). Der Anteil fachgleicher MVZ ist von $0 \%$ im Jahr 2015 auf $25 \%$ im Jahr 2017 angestiegen. MVZ konnten erst ab 2016 fachgleich gegründet werden.

\section{Zusammenfassung der Ergebnisse}

Die Angebotsstrukturen der vertragsärztlichen Versorgung in Deutschland sind durch eine sehr weitreichende Fragmentierung bei lediglich geringer und langsamer Konzentrationsdynamik geprägt. 2018 versorgten insgesamt 139393 Fachärzte/-innen der vertragsärztlichen Versorgung in insgesamt 80811 voneinander unabhängigen VO. Rund ein Viertel der VO entfielen auf die kooperativen Rechtsformen GP und MVZ, dominierende Rechtsform war die Kleinstorganisation EP mit rund $75 \%$ aller VO. Im durchschnitt-

- Tab. 1 Absolute und relative Häufigkeiten vertragsärztlicher Versorgungsorganisationen gesamt und nach Rechtsformen, über beide Versorgungsbereiche und getrennt nach hausärztlichem und fachärztlichen Versorgungsbereichen ** für 2010, 2014 und 2018.

\begin{tabular}{|c|c|c|c|c|c|c|c|}
\hline \multirow[b]{2}{*}{ Anzahl Versorgungsorganisationen } & \multicolumn{2}{|c|}{2010} & \multicolumn{2}{|c|}{2014} & \multicolumn{2}{|c|}{2018} & \multirow{2}{*}{$\begin{array}{l}\Delta 2010 / 2018 \\
\text { in } \%\end{array}$} \\
\hline & abs. & in $\%$ & abs. & in \% & abs. & in \% & \\
\hline \multicolumn{8}{|l|}{$\begin{array}{l}\text { des hausärztlichen und fachärztlichen } \\
\text { Versorgungsbereichs }\end{array}$} \\
\hline Einzelpraxen & 67022 & $76 \%$ & 63513 & $75 \%$ & 59257 & $73 \%$ & $-12 \%$ \\
\hline Gemeinschaftspraxen & 20044 & $23 \%$ & 19174 & $23 \%$ & 18381 & $23 \%$ & $-8 \%$ \\
\hline MVZ & 1654 & $2 \%$ & 2073 & $2 \%$ & 3173 & $4 \%$ & $92 \%$ \\
\hline Gesamt & 88720 & $100 \%$ & 84760 & $100 \%$ & 80811 & $100 \%$ & $-9 \%$ \\
\hline \multicolumn{8}{|c|}{ des hausärztlichen Versorgungsbereichs ${ }^{* * *}$} \\
\hline Einzelpraxen & 33504 & & 31198 & & 28668 & & $-14 \%$ \\
\hline Gemeinschaftspraxen & 9558 & & 9217 & & 9023 & & $-6 \%$ \\
\hline \multicolumn{8}{|l|}{$\mathrm{MVZ}^{*}$} \\
\hline \multicolumn{8}{|c|}{ des fachärztlichen Versorgungsbereichs $* * *$} \\
\hline Einzelpraxen & 33518 & & 32315 & & 30589 & & $-9 \%$ \\
\hline Gemeinschaftspraxen & 9350 & & 9035 & & 8624 & & $-8 \%$ \\
\hline $\mathrm{MVZ}^{*}$ & & & & & & & \\
\hline \multicolumn{8}{|c|}{$\begin{array}{l}\text { MVZ: Medizinisches Versorgungszentrum. }{ }^{*} \text { Quelle [4] erlaubt keine Auswertung, wie viele der Medizinischen Versorgungszentren in hausärztlicher } \\
\text { Versorgung und/oder fachärztlicher Versorgung tätig sind. Eine Befragung des Zentralinstitut für die Kassenärztliche Versorgung aus } 2019 \text { kommt zu dem } \\
\text { Ergebnis, dass von den in } 2017 \text { zugelassenen MVZ } 7 \% \text { ausschließlich zu dem hausärztlichen und } 48 \% \text { ausschließlich zu dem fachärztlichen Versorgungs- } \\
\text { bereich gehören [28]. }{ }^{* *} \text { Die Zahl der Gemeinschaftspraxen in der differenzierten Darstellung nach Versorgungsbereich berücksichtigt nicht die bereichs- } \\
\text { übergreifenden Gemeinschaftspraxen. }{ }^{* *}{ }^{*} \text { Keine Angaben zu prozentualen Verteilungen da quellenbedingt für den hausärztlichen und fachärztlichen } \\
\text { Versorgungsbereich nur die Zahl der Einzelpraxen und Gemeinschaftspraxen, nicht jedoch der MVZ, bekannt sind. }\end{array}$} \\
\hline
\end{tabular}


lichen Ärzteteam über alle VO waren deutlich weniger als 2, in GP weniger als 3 Fachärzte/-innen tätig. Lediglich MVZ erreichten mit einer Teamstärke von 5,9 Ärzten/Ärztinnen einen höheren Wert.

Die Unterschiede in den Angebotsstrukturen nach HV und FV sind lediglich graduell. Die Teamstärke der FV-GP ist etwas höher als in HV-GP und es arbeiten insgesamt weniger Fachärzte/-innen der FV in

- Tab. 2 Durchschnittliche ärztliche Teamstärke vertragsärztlicher Versorgungsorganisationen gesamt und nach Rechtsformen, über beide Versorgungsbereiche und getrennt nach hausärztlichem und fachärztlichen Versorgungsbereichen ** für 2010, 2014 und 2018.

\begin{tabular}{|c|c|c|c|}
\hline & 2010 & 2014 & 2018 \\
\hline \multicolumn{4}{|l|}{$\begin{array}{l}\text { Durchschnittliche ärztliche } \\
\text { Teamstärke }\end{array}$} \\
\hline $\begin{array}{l}\text { in allen vertragsärztlichen } \\
\text { Organisationen }\end{array}$ & 1,4 & 1,6 & 1,7 \\
\hline in Einzelpraxen & 1,1 & 1,1 & 1,2 \\
\hline in Gemeinschaftspraxen & 2,5 & 2,7 & 2,8 \\
\hline in $M V Z$ & 5,0 & 6,2 & 5,9 \\
\hline \multicolumn{4}{|c|}{$\begin{array}{l}\text { in Organisationen der (ausschließ- } \\
\text { lich) hausärztlichen Versorgung }\end{array}$} \\
\hline in Einzelpraxen & 1,1 & 1,1 & 1,2 \\
\hline in Gemeinschaftspraxen** & 2,4 & 2,6 & 2,7 \\
\hline \multicolumn{4}{|l|}{ in $M V Z$ * } \\
\hline \multicolumn{4}{|c|}{$\begin{array}{l}\text { in Organisationen der (ausschließ- } \\
\text { lich) fachärztlichen Versorgung }\end{array}$} \\
\hline in Einzelpraxen & 1,1 & 1,1 & 1,1 \\
\hline in Gemeinschaftspraxen ** & 2,8 & 3,1 & 3,2 \\
\hline \multicolumn{4}{|l|}{ in $M V Z^{*}$} \\
\hline \multicolumn{4}{|c|}{$\begin{array}{l}\text { MVZ: Medizinische Versorgungszentren. }{ }^{*} \text { Quelle [4] erlaubt getrennte } \\
\text { Aufschlüsselung nach Bereichen nur für Einzelpraxen und Gemeinschaft- } \\
\text { spraxen. }{ }^{* *} \text { In die Berechnung der Teamstärke in Gemeinschaftspraxen in } \\
\text { der Darstellung nach Bereich sind keine versorgungsbereichsübergreifen- } \\
\text { den Gemeinschaftspraxen eingegangen. }\end{array}$} \\
\hline
\end{tabular}

Einzelpraxen und mehr in MVZ. Die Angebotsstrukturen beider Bereiche werden jedoch von EP dominiert. Die etwas größere Variabilität der bereichsbezogenen Daten legt nahe, dass die fachärztlichen Angebotsstrukturen etwas heterogener sind als die hausärztlichen.

Eine interdisziplinäre Aufstellung von VO war die Ausnahme, lediglich $17 \%$ aller GP und MVZ versorgten entsprechend. Aus anderen Quellen ist bekannt, dass einige Fächer - allen voran die methodenorientierten Fächer wie Pathologie, Laboratoriumsmedizin und Mikrobiologie, Virologie und Infektionsmedizin - überdurchschnittlich häufig eine interdisziplinäre Aufstellung wählen [8]. Insgesamt scheinen die Gründe für eine monofachliche Aufstellung zu überwiegen. Diskutiert werden dabei regulatorische und medizinisch-fachliche Aspekte, zudem schaffe die Gebührenordnung für die Vertragsärztliche Versorgung keine Anreize für interdisziplinäre Kooperation innerhalb einer VO [9].

Unter Annahme einer konstanten Veränderungsgeschwindigkeit werden die Angebotsstrukturen in Deutschland perspektivisch noch lang von kleinsten und kleinen VO geprägt bleiben. Der hohe Grad der Fragmentierung reduzierte sich trotz gut dokumentierter sich ändernder Präferenzen junger Ärzte/-innen (Arbeit im Team, Möglichkeit zur Anstellung $[10,11]$ ) und der steigenden Zahl angestellter Fachärzte und -innen [12] nur geringgradig. MVZ wiesen zwar größere Teamstärke und mehr Interdisziplinarität auf, sie waren aber auch 14 Jahre nach ihrer Einführung mit $4 \%$ aller VO nur ein Randphänomen. MVZ entwickelten sich auch nach Arztzahlen eher moderat: die Zahl der in MVZ beschäftigten Ärzte/-innen nahm zwischen 2010 und 2018 pro Jahr um rund 1300 zu (zum Vergleich: 2018 wurden 8652 Ärzte/-innen neu vertragsärztlich tätig [12]).

\section{Diskussion}

Im Sinne der Limitation der Auswertung sei angemerkt, dass die Sekundärdatenquellen der KBV uneinheitliche Kategorisierungen verwenden, sodass Auswertungen unter Kombination verschiede-

- Tab. 3 Absolute und relative Häufigkeit von Fachärzten/-innen gesamt und nach Rechtsformen, über beide Versorgungsbereiche und getrennt nach hausärztlichem und fachärztlichen Versorgungsbereichen für 2010, 2014 und 2018.

\begin{tabular}{|c|c|c|c|c|c|c|c|}
\hline \multirow[b]{2}{*}{ Anzahl Fachärzte/Fachärztinnen } & \multicolumn{2}{|c|}{2010} & \multicolumn{2}{|c|}{2014} & \multicolumn{2}{|c|}{2018} & \multirow[t]{2}{*}{$\Delta 2010 / 2018$} \\
\hline & abs. & in $\%$ & abs. & in $\%$ & abs. & in $\%$ & \\
\hline in allen vertragsärztlichen Organisationen & 128450 & & 134152 & & 139393 & & $9 \%$ \\
\hline \multicolumn{8}{|l|}{ Davon } \\
\hline in Einzelpraxen & 70916 & $55 \%$ & 69957 & $52 \%$ & 68361 & $49 \%$ & $-4 \%$ \\
\hline in Gemeinschaftspraxen & 49208 & $38 \%$ & 51240 & $38 \%$ & 52207 & $37 \%$ & $6 \%$ \\
\hline in MVZ & 8326 & $6 \%$ & 12955 & $10 \%$ & 18825 & $14 \%$ & $126 \%$ \\
\hline in der hausärztlichen Versorgung, gesamt & 60573 & & 60738 & & 61845 & & $2 \%$ \\
\hline \multicolumn{8}{|l|}{ davon } \\
\hline in Einzelpraxen & 35631 & $59 \%$ & 34734 & $57 \%$ & 33949 & $55 \%$ & $-5 \%$ \\
\hline in Gemeinschaftspraxen & 23224 & $38 \%$ & 23592 & $39 \%$ & 24298 & $39 \%$ & $5 \%$ \\
\hline in MVZ & 1718 & $3 \%$ & 2412 & $4 \%$ & 3598 & $6 \%$ & $109 \%$ \\
\hline in der fachärztlichen Versorgung, gesamt & 67877 & & 73414 & & 77548 & & $14 \%$ \\
\hline \multicolumn{8}{|l|}{ davon } \\
\hline in Einzelpraxen & 35285 & $52 \%$ & 35223 & $48 \%$ & 34412 & $44 \%$ & $-2 \%$ \\
\hline in Gemeinschaftspraxen & 25984 & $38 \%$ & 27648 & $38 \%$ & 27909 & $36 \%$ & $7 \%$ \\
\hline in MVZ & 6608 & $10 \%$ & 10543 & $14 \%$ & 15227 & $20 \%$ & $130 \%$ \\
\hline
\end{tabular}




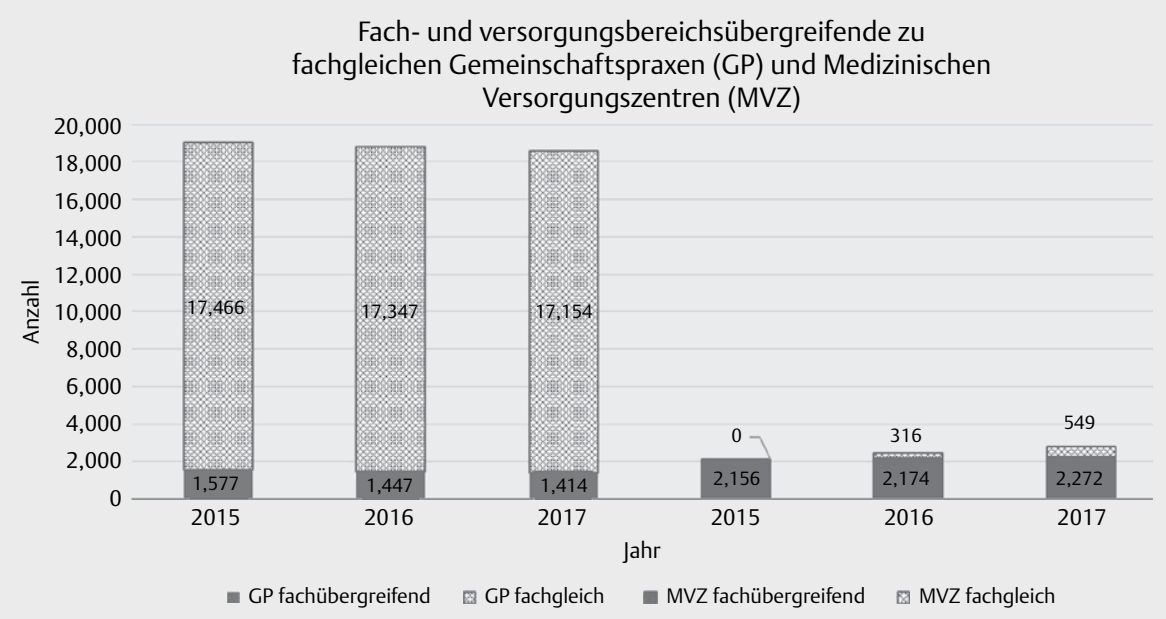

- Abb. 1 Anzahl fach- bzw. versorgungsbereichsübergreifender und fachgleicher Gemeinschaftspraxen (GP) und Medizinischer Versorgungszentren (MVZ), 2015 bis 2017.

ner Quellen nur teilweise möglich sind. Für eine Beschreibung der Angebotsstrukturen scheint es jedoch gravierender, dass zu VO ausschließlich entlang von Rechtsformen berichtet wird, Daten zu Verteilung anderer Merkmale fehlen. Es ist jedoch davon auszugehen, dass potentiell versorgungsrelevante Unterschiede sich nicht (alleine) in der Rechtsform manifestieren.

\section{Abgleich mit ambulanten Angebotsstrukturen anderer dezentraler Gesundheitssysteme}

Die Online-Datenbank des Commonwealth Fund mit textbasierten halb standardisierten Profilinformationen zu Gesundheitswesen, u. a. auch zu „Delivery system“ (Art der Bereitstellung medizinischer Leistungen) wurde für den Abgleich von ambulanten Angebotsstrukturen verwendet [13]. Berücksichtigt wurden nur Gesundheitswesen aus Nordamerika und Europa, deren ambulante Versorgung ganz oder überwiegend dezentral in von Kostenträgern bzw. staatlicher Administration unabhängigen VO und ganz oder überwiegend von selbstständigen Fachärzten/-innen erbracht wird. Diese Kriterien erfüllten die Länder Dänemark, England, Frankreich, Italien, Kanada, Niederlande, Norwegen, Schweiz und USA ( $>$ Tab. 4).

Bezogen auf die Merkmale ,Häufigkeit von Einzelpraxen` und ,Teamstärke‘ weisen die deutschen Angebotsstrukturen eine eher größere Kleinteiligkeit auf als andere Gesundheitssysteme. Eine vergleichbar hohe Zahl an Einzelpraxen wie in Deutschland wird noch aus Frankreich (50\% der „General practicioner“ in EP) und der Schweiz (54\% der „General practicioner“ und „Specialists“ in EP) berichtet. In anderen Ländern mit Daten zu diesem Merkmal sind mindestens 2/3 der hausärztlich tätigen Fachärzte/-innen in GP tätig. Eine weitere Besonderheit: In Deutschland findet auch die fachärztliche Versorgung überwiegend in Einzelpraxen statt. Auch die durchschnittliche Teamstärke ist in Deutschland gering, ähnliche niedrige Werte in kooperativen VO werden nur aus Dänemark und Frankreich berichtet. Aus anderen Ländern werden höhere Durchschnittswerte (Schweiz 4,2, England 4,6 (eigene Berechnung auf Basis der Angaben in der Quelle)) oder höhere Bandbreiten in den Arztzahlen berichtet.
In anderen Ländern mit kleinteiligen ambulanten Angebotsstrukturen zielen staatliche Initiativen auf stärkere Konzentration. Das gilt z. B. für die Schweiz [14], Frankreich („loi de modernisation du système de santé“ aus 2016) und Dänemark [15]; die OECD gibt explizit der „Group Practice“ den Vorzug gegenüber „Solo Practices“ [16]. Ein entsprechendes Politikziel ist in der deutschen Gesundheitsgesetzgebung nicht zu erkennen; hier liegt der Fokus auf (freiwilligen) Kooperationsmöglichkeiten unabhängiger VO.

Eine fachübergreifende Aufstellung von VO scheint in der überwiegenden Zahl der Länder zwar angestrebt zu werden, die Länderberichte liefern jedoch mit Ausnahme von Kanada (19\% der Hausärzte/-innen in interprofessionellen Teams) keine Häufigkeitsangaben.

\section{Ambulante Angebotsstrukturen, organisationsbezogene Versorgungsforschung und Versorgungssteuerung}

In den letzten Jahren hat sich das Spektrum von VO z. B. nach Anzahl Standorten, Leistungsspektrum, Versorgungstiefe, Spezialisierungsgrad, Tätigkeitsformen der Ärzte/-innen, Trägermodellen, Beteiligungskapital usw. auch innerhalb einzelner Rechtsformen ausdifferenziert, Fachspezifika haben zusätzlich Einfluss. Im Interesse eines besseren Verständnisses und damit auch einer gezielteren Versorgungssteuerung wäre Forschung zu organisationsbezogenen Merkmalen und eventuellen Wechselwirkungen mit Versorgungsgeschehen wünschenswert.

Bislang wurden jedoch wenig Studienergebnisse zu den Wechselwirkungen veröffentlicht [17-22]. Die Diskussion wird derzeit vorrangig in den USA geführt, Motoren sind die Möglichkeit zur Gründung von Accountable Care Organizations (ACO) und der wachsende Beitrag von fachfremden Investoren [23]. In systematischen Auswertungen ist die Evidenz zur Effektivität entlang von Indikatoren wie Versorgungsqualität, Ergebnisqualität, Inanspruchnahme, Gesundheitsausgaben und Patientenzufriedenheit (zumindest bislang) partiell heterogen. Eine Ursache für die wenig entwickelte Studienlage dürfte die hohe Variabilität der VO und deren geringer Standardisierungsgrad sein. 


\begin{tabular}{|c|c|c|c|c|c|c|c|c|c|c|}
\hline \multicolumn{3}{|c|}{ 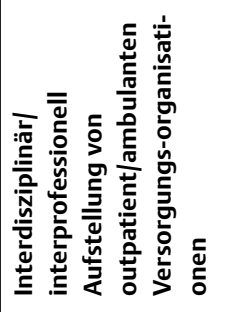 } & 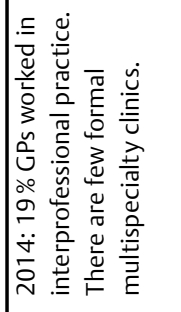 & 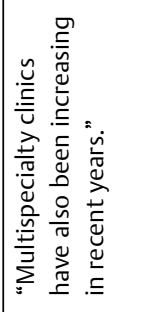 & & 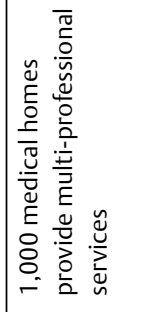 & 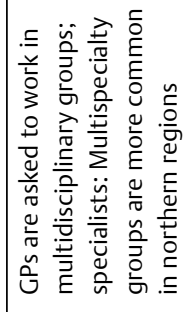 & 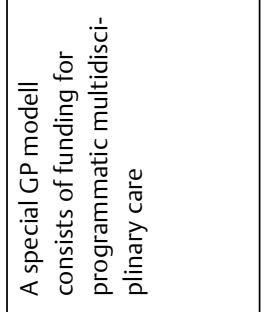 & 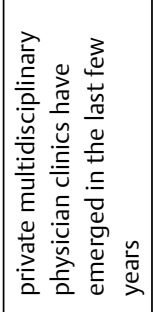 & \\
\hline \multicolumn{3}{|c|}{ 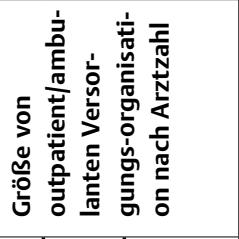 } & & 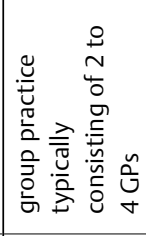 & 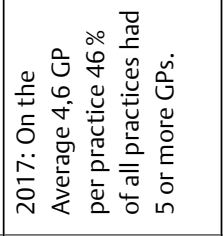 & 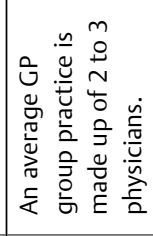 & 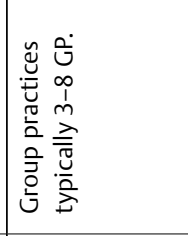 & 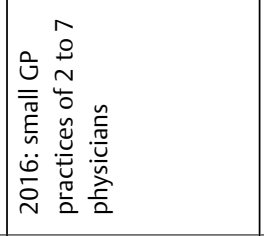 & 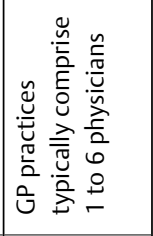 & 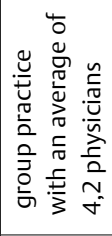 \\
\hline \multirow{4}{*}{ 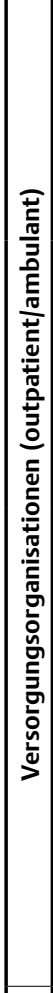 } & 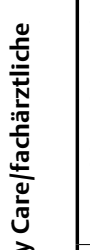 & 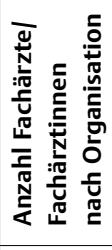 & & & & 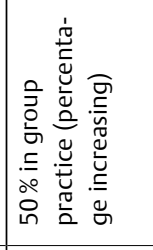 & & & & 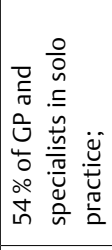 \\
\hline & 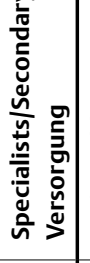 & 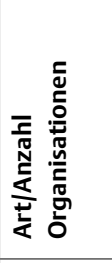 & 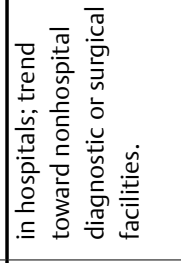 & 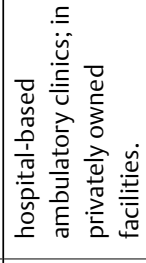 &  & 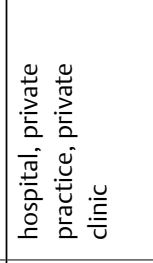 & 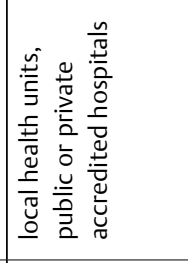 & 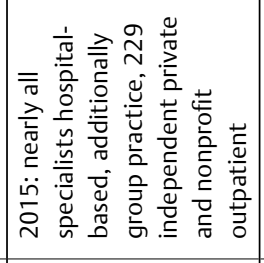 & 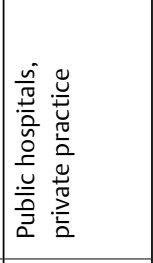 & 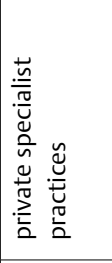 \\
\hline & \multirow{2}{*}{ 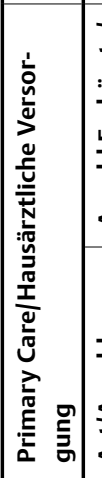 } & 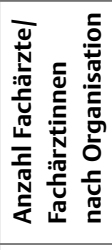 & 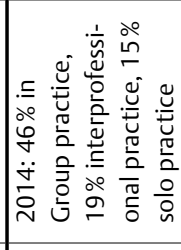 & & & 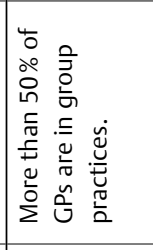 & 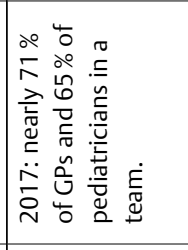 &  & & 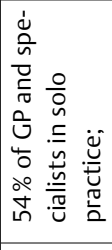 \\
\hline & & 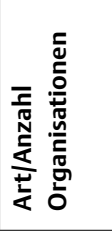 & & 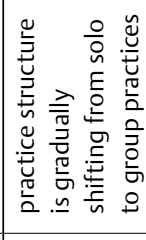 &  & & & & 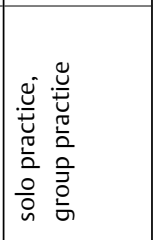 & 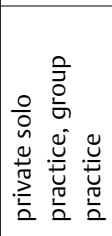 \\
\hline \multirow{2}{*}{ 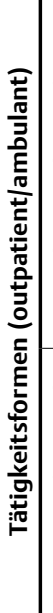 } & \multicolumn{2}{|l|}{ 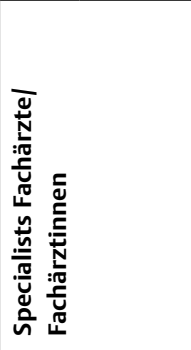 } & 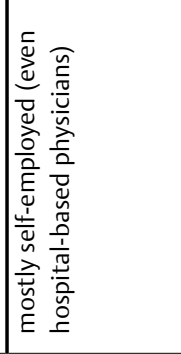 & 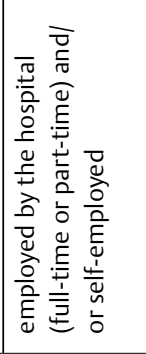 & 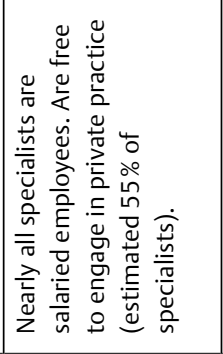 & 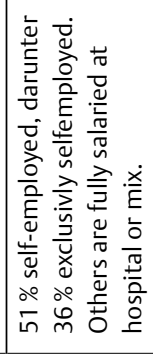 & 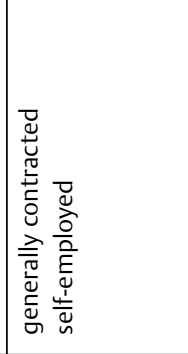 & 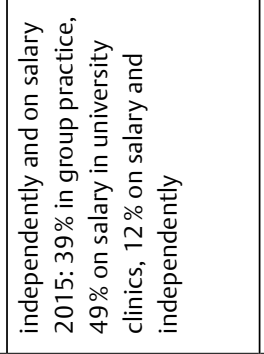 & 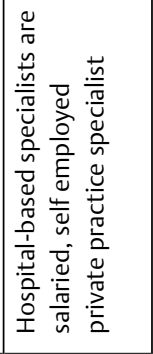 & 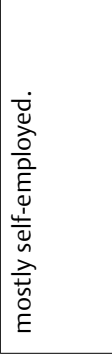 \\
\hline & \multicolumn{2}{|c|}{ 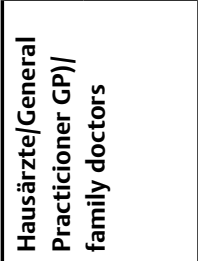 } & 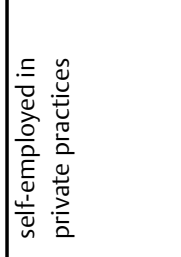 & 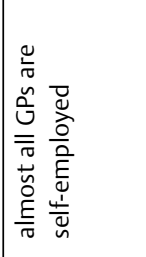 & 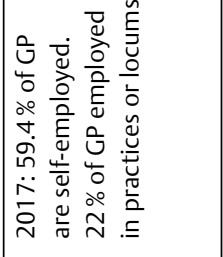 & 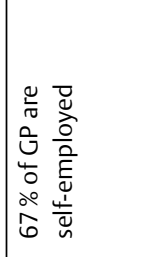 & 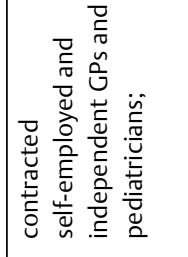 & 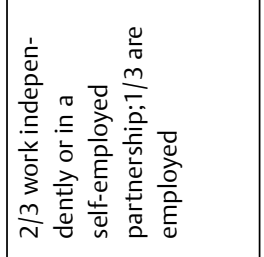 & 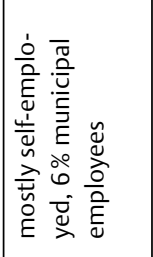 & 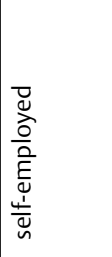 \\
\hline & & & 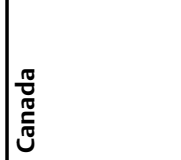 & 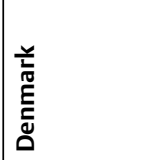 & 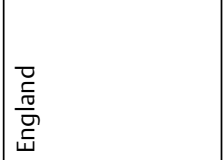 & \begin{tabular}{|l} 
竞 \\
胥
\end{tabular} & \pm & 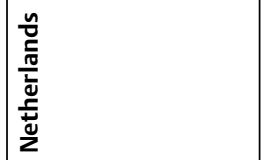 & \begin{tabular}{|l} 
ते \\
3. \\
20
\end{tabular} & 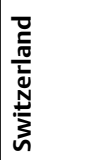 \\
\hline
\end{tabular}




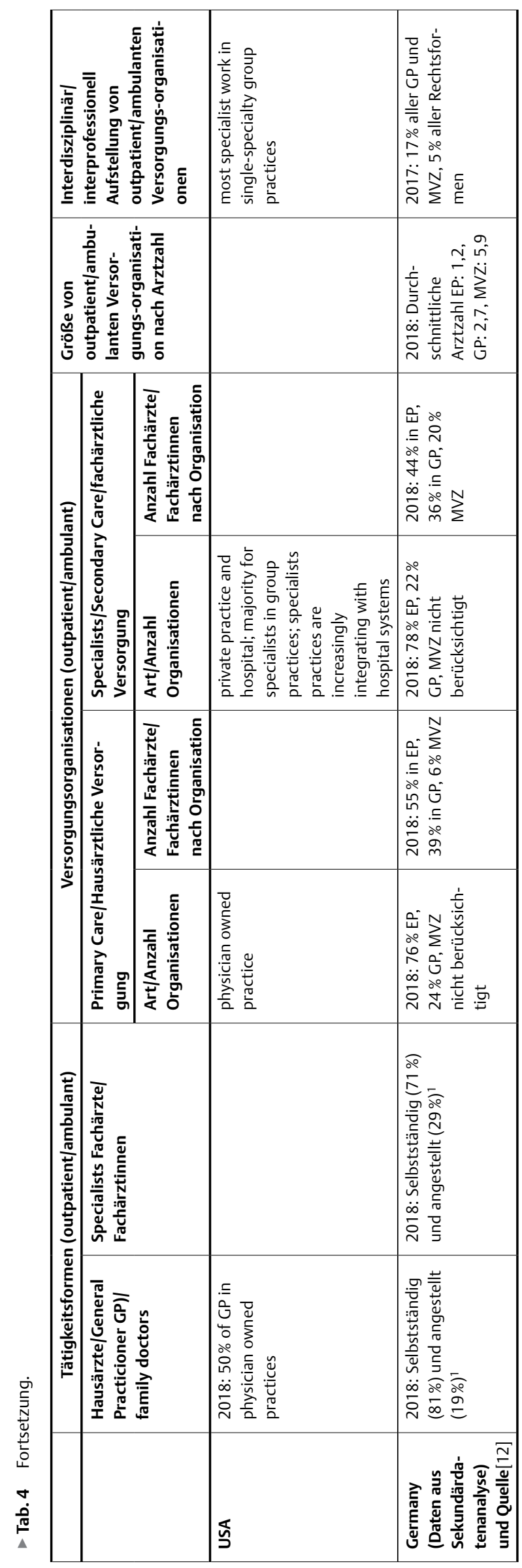

Im Sinne einer gezielteren Versorgungssteuerung ist eine Auseinandersetzung mit den Wechselwirkungen von Merkmalen von VO und Versorgungsparametern dessen ungeachtet wünschenswert. Ein Beispiel soll das illustrieren: Allgemein beklagt wird, dass die medizinische Versorgung auf dem Land unzureichend sei [3, 24]. Im Vorfeld einer gesundheitspolitischen Maßnahme wäre es sinnvoll zu wissen, ob eine überproportional häufige Versorgung an peripheren Standorten mit bestimmten Merkmalen von VO (z. B. nach Größe, Leistungsspektrum oder nach Filialisierung) oder mit bestimmten Tätigkeitsformen von Ärzten/-innen (angestellte vs. selbstständige Ärzte) vergesellschaftet ist. In Kenntnis von Zusammenhängen ließen sich Ansatzpunkte für eine gezieltere Versorgungssteuerung ableiten [25].

Eine in diesem Sinne verstandene organisationsbezogene Versorgungsforschung setzt eine ausreichende Datenverfügbarkeit voraus. Die Analysemöglichkeiten zu der stationären Versorgung sind dank der verpflichtenden Qualitätsberichte nach § 136b Abs. 1 Satz 1 Nr. 3 Sozialgesetzbuch (SGB) V besser. Krankenhäusern können entlang von Merkmalen wie z. B. Versorgungsebene, Trägerschaft oder Größe nach Betten- oder Abteilungszahl typisiert werden [26]. Organisationsbezogene Merkmale von Krankenhäusern werden bereits als Aufgreifkriterium für versorgungssteuernde Maßnahmen herangezogen (Beispiel: „Zentren für besondere Aufgaben“ § 2 Abs. 2 Gesetz über die Entgelte für voll- und teilstationäre Krankenhausleistungen). Eine ausreichend differenzierte Berichtssystematik wäre für die vertragsärztliche Versorgung ebenfalls wünschenswert, muss aber den Besonderheiten dieses Versorgungsbereichs mit über 80000 einzelnen VO Rechnung tragen. Als Basis kommen von den Kassenärztlichen Vereinigungen (KVen) erfasste Daten wie z. B. die lebenslange Arztnummer oder die Betriebsstättennummer in Frage [27].

\section{FAZIT}

Auf Basis von Sekundärdatenquellen zeigt sich eine von Kleinteiligkeit geprägte überwiegend monofachlich aufgestellte vertragsärztliche Angebotsstruktur mit nur leichter Tendenz zur Konzentration. Die Einzelpraxis dominiert sowohl die hausärztliche als auch die fachärztliche Versorgung. MVZ sind ein Randphänomen, das in der fachärztlichen Versorgung etwas stärker verankert ist als in der hausärztlichen. Die Fragmentierung nach Anzahl Versorgungsorganisationen und durchschnittlicher Teamstärke ist in Deutschland ausgeprägter als in anderen dezentral organisierten Gesundheitssystemen. Ein Grund dafür ist, dass der deutsche Gesetzgeber den Fokus auf freiwillige Kooperation selbstständiger Einheiten legt und eine stärkere Integration von nach Arztzahlen größeren Versorgungsorganisationen nicht explizit begünstigt. Die verfügbare Sekundärdatenbasis mit summarischen Angaben nach Rechtsformen ist für eine differenzierte Analyse von Wechselwirkungen zwischen Merkmalen von Versorgungsorganisationen und Versorgungsgeschehen nicht ausreichend. Eine umfangreichere Datenbereitstellung - z. B. auf Basis von Abrechnungsdaten - und größere Transparenz wären im Sinne eines besseren Verständnisses und als Basis für versorgungssteuernde Eingriffe wünschenswert. 


\section{Interessenkonflikt}

Die Autorinnen/Autoren geben an, dass kein Interessenkonflikt besteht.

\section{Literatur}

[1] Ansmann L, Albert U-S, Auer R et al. DNVF-Memorandum III - Methoden für die Versorgungsforschung, Teil 4 - Konzept und Methoden der organisationsbezogenen Versorgungsforschung: Kurzfassung. Das Gesundheitswesen 2019; 81: 220-224. doi:10.1055/a-0862-0407

[2] Sachverständigenrat zur Begutachtung der Entwicklung im Gesundheitswesen Kooperation und Verantwortung - Voraussetzungen einer zielorientierten Gesundheitsversorgung. In: Sachverständigenrat zur Begutachtung der Entwicklung im Gesundheitswesen ed, Gutachten 2007. Bonn: 2007

[3] Kuhn B, Steinhäuser ], Eberhard S et al. Die Rolle von niedersächsischen Kommunen fur die zukunftige ärztliche Versorgung - Eine Befragung der Bürgermeister und Landräte. Gesundheitswesen 2018; 80: 711-718. doi:10.1055/s-0042-121602

[4] Kassenärztliche Bundesvereinigung. Anzahl Praxen nach Praxisart, 2008-2018. Berlin: Kassenärztliche Bundesvereinigung; 2019: https:// gesundheitsdaten.kbv.de/cms/html/17020.php

[5] Kassenärztliche Bundesvereinigung. Anzahl Ärzte in Praxen nach Art der Praxis, 2010-2018. Berlin: Kassenärztliche Bundesvereinigung; 2019: https://gesundheitsdaten.kbv.de/cms/html/17019.php

[6] Kassenärztliche Bundesvereinigung. Statistische Informationen aus dem Bundesarztregister Bundesgebiet insgesamt. Berlin: Kassenärztliche Bundesvereinigung; jährlich: https://www.kbv.de/html/ bundesarztregister.php

[7] Bundesregierung. Antwort der Bundesregierung auf die Kleine Anfrage der Abgeordneten Dr. Achim Kessler, Susanne Ferschl, Klaus Ernst, weiterer Abgeordneter und der Fraktion DIE LINKE. Kapitalinteressen bei der Übernahme von Medizinischen Versorgungszentren. In: Bundesgesundheitsministerium. ed. Berlin: Bundesdruckerei; 2018

[8] Ansmann L, Baumann W, Gostomzyk J et al. DNVF-Memorandum III Methoden für die Versorgungsforschung, Teil 4 - Konzept und Methoden der organisationsbezogenen Versorgungsforschung. Kapitel 1 - Definition und Konzept der organisationsbezogenen Versorgungsforschung. Das Gesundheitswesen 2019; 81: 64-71. doi:10.1055/a-0862-0527

[9] Müller S. Förderung von Kooperation: Theorie trifft Realität. Arzt und Krankenhaus 2013; 86: 325-329

[10] Hahn U, Müller T, Awe B et al. Angestellte Ärzte in der ambulanten Versorgung - Trends, Status Quo und Auswirkungen. Gesundheitsökonomie und Qualitätsmanagement 2017; 22: 93-103. doi:http:// dx.doi.org/10.1055/s-0042-113352

[11] Drager S, Gibis B, Jacob R et al. Zur Situation der angestellten Ärztinnen und Ärzte im ambulanten Sektor in Deutschland: Ergebnisse einer empirischen Studie. Das Gesundheitswesen 2019; Online ahead of print. doi:10.1055/a-1005-7262

[12] Kassenärztliche Bundesvereinigung. Statistische Informationen aus dem Bundesarztregister Bundesgebiet insgesamt, Stand: 31.12.2018. Berlin: Kassenärztliche Bundesvereinigung; 2019: https://www.kbv.de/ media/sp/2018_12_31_BAR_Statistik.pdf

[13] Tikkanen R, Osborn R, Mossialos E et al. How is the delivery system organized and how are providers paid? In: INTERNATIONAL HEALTH CARE SYSTEM PROFILES. Washington: The Commonwealth Fund; 2020: https://www.commonwealthfund.org/international-healthpolicy-center/system-features/how-delivery-system-organized-andhow-are
[14] Doty MM, Tikkanen R, Shah A et al. Primary Care Physicians' Role In Coordinating Medical And Health-Related Social Needs In Eleven Countries. Health Aff (Millwood) 2020; 39: 115-123. doi:10.1377| hlthaff.2019.01088

[15] Voit D. Die neuen Qualitätsziele in Dänemark; Impulse für Deutschland? Das Krankenhaus 2018; 9.2018 820-824

[16] OECD - Organisation for Economic Co-operation and Development. Realising the Potential of Primary Health Care. Im Internet: https:// www.oecd-ilibrary.org/sites/a92adee4-en/index.html?itemld = / content/publication/a92adee4-en Stand: 2020/10/20 doi:https://doi. org/10.1787/a92adee4-en

[17] Tollen L. Physician Organization in Relation to Quality an Efficiency of Care: a Synthesis of Recent Literatur Commonwealth Fund. 2008; April 2008

[18] Damiani G, Silvestrini G, Federico B et al. A systematic review on the effectiveness of group versus single-handed practice. Health Policy 2013; 113: 180-187. doi:10.1016/j.healthpol.2013.07.008

[19] Ehlert A. Kooperationsverhalten niedergelassener Ärzte in Norddeutschland: Statistische Analyse der Einflussfaktoren. Gesundheitsökonomie und Qualitätsmanagement 2017; 22: 290-296

[20] Hildebrandt H, Pimperl A, Schulte T et al. Triple Aim - Evaluation in der Integrierten Versorgung Gesundes Kinzigtal - Gesundheitszustand, Versorgungserleben und Wirtschaftlichkeit. Bundesgesundheitsblatt Gesundheitsforschung Gesundheitsschutz 2015; 58: 383-392. doi:10.1007/s00103-015-2120-y

[21] Laux G. Hausarztzentrierte Versorgung - Evaluationsergebnisse aus Baden-Württemberg. In: Pundt J, Hrsg. Vernetzte Versorgung - Lösung für Qualitäts- und Effizienzprobleme in der Gesundheitswirtschaft. Bremen: Apollon Hochschule für Gesundheitswirtschaft; 2017: 237-258

[22] Zentner A, Velasco Garrido M, Busse R. Macht der Hausarzt als Lotse die Gesundheitsversorgung wirklich besser und billiger? Ein systematischer Review zum Konzept Gatekeeping. Gesundheitswesen 2010; 72: e38-e44. doi:10.1055/s-0029-1234126

[23] Zhu JM, Hua LM, Polsky D. Private Equity Acquisitions of Physician Medical Groups Across Specialties, 2013-2016. JAMA 2020; 323 : 663-665. doi:10.1001/jama.2019.21844

[24] Sachverständigenrat zur Begutachtung der Entwicklung im Gesundheitswesen Bedarfsgerechte Versorgung Perspektiven für ländliche Regionen und ausgewählte Leistungsbereiche.In Sachverständigenrat zur Begutachtung der Entwicklung im Gesundheitswesen, Gutachten 2014. Bonn/Berlin: 2014

[25] Hahn U, Awe B, Koch J et al. Intersektorale augenmedizinische Facharztzentren und Versorgung auf dem Land. Gesundheitsökonomie und Qualitätsmanagement 2019; 24: 69-77. doi:10.1055/a-0590-7298

[26] Augurzky B, Krolop S, Mensen A et al. Das Ende des Wachstums. In: Medhochzwei ed, Krankenhaus Rating Report 2019. Wiesbaden: Medhochzwei; 2019

[27] Grobe TG, Dräther H Ambulante ärztliche Versorgung. In: Swart E, Ihle P, Gothe $\mathrm{H}$ et al., Hrsg. Routinedaten im Gesundheitswesen; Handbuch Sekundärdatenanalse: Grandlagen, Methoden und Perspektiven. Bern: Verlag Hans Huber; 2015: 43-62

[28] Zentralinstitut für die kassenärztliche Versorgung in Deutschland Zi-MVZ-Panel, Berichtsjahr 2017. In: MVZ Fachinformation. Berlin: 2020: https://www.zi.de/fileadmin/images/content/PDFs_alle/ Fachinfo_MVZ_2020_Final.pdf 